NBSIR 88-3091

\title{
GROUP INDEX AND \\ TIME DELAY MEASUREMENTS \\ OF A STANDARD REFERENCE FIBER
}

\section{B. L. Danielson \\ C. D. Whittenberg}

\section{FHE CRPY \\ DO MT REMOIE}

National Bureau of Standards

U.S. Department of Commerce

Boulder, Colorado 80303-3328

July 1988

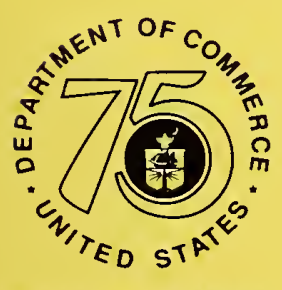



NBSIR 88-3091

\section{GROUP INDEX AND \\ TIME DELAY MEASUREMENTS \\ OF A STANDARD REFERENCE FIBER}

\section{B. L. Danielson \\ C. D. Whittenberg}

Electromagnetic Technology Division

Center for Electronics and Electrical Engineering

National Engineering Laboratory

National Bureau of Standards

Boulder, Colorado 80303-3328

July 1988

Sponsored in part by

Department of the Navy

Naval Weapons Station/Seal Beach

Corona, California 91720-5000

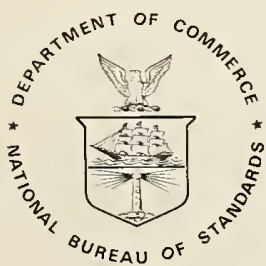

U.S. DEPARTMENT OF COMMERCE, C. William Verity, Secretary NATIONAL BUREAU OF STANDARDS, Ernest Ambler, Director 



\section{Contents}

Page

1. Introduction $\ldots \ldots \ldots \ldots \ldots \ldots \ldots \ldots \ldots \ldots \ldots \ldots \ldots \ldots \ldots$

2. Group Index Measurements .................... 3

2.1 Theory of the Scanning Interferometer ........... 4

3. Time Delay Measurements .................... 5

4. Error Analysis ........................ 5

4.1 Group Index Errors ..................... 6

4.2 Pulse Time-of-Flight Errors ................ 8

4.3 Total Length Uncertainty for Long Fibers ........... 9

5. OTDR Calibration ......................... 9

6. Conclusion ............................. 10

7. References ............................. 10 



\title{
Group Index and Time Delay Measurements of a Standard Reference Fiber
}

B. L. Danielson and C. D. Whittenberg National Bureau of Standards Boulder, Colorado 80303-3328

\begin{abstract}
We describe measurement techniques for establishing a standard reference fiber with well characterized group index and time o1 group delay. Evaluation of an interferometric method indicates that fiber group index can be determined with a total estimated uncertainty of about 0.03 percent in small samples. Group delay of the reference fiber was measured with an overall uncertainty less than 0.004 percent in a $7 \mathrm{~km}$ waveguide. We discuss the application of a standard reference fiber to calibration of the distance measurement accuracy of an optical time-domain reflectometer (OTDR).
\end{abstract}

Key words: calibration procedures; optical delay line; optical fiber group index; optical fiber group velocity; optical timedomain reflectometer; OTDR; standard reference fiber; standard test fiber

\section{Introduction}

A standard reference fiber (SRF) is a commercially available lightguide which has been well characterized for selected transmission properties at certain wavelengths [1]. Such a fiber can be used as an interim standard reference material for application in calibrating test instruments such as the optical time-domain reflectometer (OTDR) and also for evaluation of the accuracy of different measurement techniques through interlaboratory comparisons. 
This report is primarily concerned with the development of techniques for precision length determinations of single-mode optical fibers at a wavelength of $1300 \mathrm{~nm}$. We also discuss the application of these techniques to the calibration of distance measurement accuracy for the optical time-domain reflectometer.

Two methods can be used to measure fibers that are several kilometers in length. Winding machines used for wrapping fiber on spools usually have provisions for distance readout. Also, length can be inferred from transmitted time of flight of an optical pulse. The latter approach requires a knowledge of fiber group index and delay time of an optical pulse of short duration. Of the two methods, we think that the optical means are not only more versatile and controllable, but more accurate as well. We will, therefore, only consider optical length determinations here.

Fiber length $\mathrm{L}$ can be calculated from the relation,

$$
\mathrm{L}=\frac{c \mathrm{~T}_{\mathrm{d}}(\lambda)}{\mathrm{N}(\lambda)} \text {, }
$$

where $T_{d}(\lambda)$ is the time delay or group delay of an optical pulse transmitted one way through the test fiber at the wavelength $\lambda$, and $c / N(\lambda)$ the group velocity. Here $c$ is the velocity of light and $N(\lambda)$ is the effective group index of refraction of the fiber defined as a ratio of the speed of light in a vacuum to the speed of an optical pulse in the lightguide, when the pulse has a spectral content spread over a finite wavelength interval [2]. Independent optical measurements of $N(\lambda)$ and $T_{d}(\lambda)$ at a common wavelength are necessary and sufficient for determining a unique value of the physical length of the fiber. 


\section{Group Index Measurements}

The group index (or group velocity) of a fiber is not only a function of wavelength but will also vary from fiber to fiber depending on the specific profile design as well as manufacturing details. For the most accurate length determinations, the group index of the individual fiber or cable under test must be known. The effective group velocity of a cable may be different from the constituent fibers due to an excess fiber path designed for stress relief in the cable. Group index data may sometimes be available from the manufacturer $[3,4]$. Experimentally, the effective group index may be obtained from eq (1) by an inverse process where propagation delay information is available on a fiber of known length. In principle, any of the techniques for measuring dispersion can be used for this purpose [5]. A key problem in this connection involves establishing accurate values for length.

We have chosen here to evaluate a new interferometric technique [6] which is simpler and uses shorter samples than those reported in other interferometric methods. The technique is illustrated in figure 1. The procedure is as follows:

(1) The physical length of the fiber $L_{p}$ is measured by placing a fiber sample in a vacuum chuck mounted on a translation stage, and noting the distance on the translation micrometer required to align either end of the fiber with crosshairs in the microscope eye piece. The length of the sample in these experiments was limited by the maximum travel of the micrometer to about $50 \mathrm{~mm}$.

(2) The optical length $\mathrm{L}_{\mathrm{opt}}$ is measured from observations of the change in air path in the reference arm between visibility maxima in the interferograms reflected from the two ends of the fiber. Typical signatures are illustrated in figure 2.

(3) The group index is then calculated from the relation 


$$
N=\frac{L_{o p t}}{L_{p}}
$$

\subsection{Theory of the Scanning Interferometer}

The complete theory of the asymmetric scanning Michelson interferometer is given in standard references by Bell [7] and Chamberlain [8]. Chapter 8 of [7] shows that the shift in the peak of the interferogram (the grand maximum) occasioned by insertion of a sample with index of refraction $n(\lambda)$ in an otherwise balanced interferometer is given by

$$
D=(\bar{n}-1) L_{p}
$$

where $\bar{n}$ is some suitable average index of refraction. However, the location of the maximum is given by the stationary phase condition,

$$
\frac{d}{d \lambda}\left(\frac{2 \pi}{\lambda}[\mathrm{n}-1] \mathrm{L}_{\mathrm{p}}-\mathrm{D}\right)=0
$$

This is just the condition which defines the group index [1],

$$
N(\lambda)=n-\lambda \frac{d n}{d \lambda}
$$

We conclude that the excess optical path due to the sample is

$$
D=(N-1) L_{p},
$$

which is equivalent to eq (2). 


\section{Time Delay Measurements}

Lightguides can be used as precise optical delay lines [9]. In some signal processing systems the value of this delay is critical [10]. In order to minimize wavelength dependence, we have measured $\mathrm{T}_{\mathrm{d}}$ at the zero-dispersion wavelength $\lambda_{0}$ (around $1300 \mathrm{~nm}$ for dispersion unshifted fibers). The pulse time-of-flight was obtained with a conventional digital delay generator as illustrated in figure 3. The full width at half maximum of the optical pulse, convolved with the oscilloscope response, is about 600 ns (fig. 4). The pulse transit time in the SRF was obtained by adjusting the electronic delay of the digital delay generator to equal the optical delay in the test fiber. The equality of delays was established by aligning the pulse with a fiducial point on the oscilloscope set from a short jumper fiber.

\section{Error Analysis}

We use Gauss's law of propagation of error to estimate the standard deviation of the total uncertainty in the quantities we wish to measure (group index, time delay, and length). In this case the total uncertainty, $\sigma_{\mathrm{N}}$, is a function of the errors in several observables, $\sigma_{i}$, and we can write

$$
\left(\frac{\sigma_{\mathrm{N}}}{\mathrm{N}}\right)^{2}=\sum\left(\frac{\sigma_{\mathrm{i}}}{\mathrm{x}_{\mathrm{i}}}\right)^{2}
$$

where $\sigma_{i}$ is the standard deviation of each uncorrelated observable $x_{i}$. Similar expressions are assumed to hold for the time delay and total SRF length. For simplicity we will not distinguish between systematic and random errors in this analysis. 


\subsection{Group Index Errors}

Systematic errors inherent in the measurement of fiber sample length $\mathrm{L}_{\mathrm{p}}$ include those arising from end wedge angles and uncertainties in determining the optical features which indicate the fiber end. Random errors occur since the microscope crosshairs can not be precisely aligned with the ends. The end angle, as determined from an interference microscope [11], was kept less than $1^{\circ}$. This insured that uncertainties due to this source (considering both ends) were less than $2 \mu \mathrm{m}$. The precision of repeated measurements was about $7 \mu \mathrm{m}$. The total uncertainty in $\mathrm{L}_{\mathrm{p}}$ was estimated to have a onesigma value of about $1.8 \times 10^{-4}$.

There are similar uncertainties in determining the optical length $\mathrm{L}_{\text {opt }}$. The stepping motor translation stage was compared directly with the micrometer by moving the micrometer screw in one direction and the stepping motor a nominally equal amount in the opposite direction and noting any discrepancies in fringe location. The stepping motor drive did not completely agree with the micrometer, and a small correction was added to the reading indicated on the motor drive unit $\left(1.9 \times 10^{-4}\right)$. Again, the micrometer was assumed to have no systematic errors. A small hystersis in the stepping motor drive could be eliminated by maintaining a consistent direction of travel for all observations. The precision of location of the peak fringe was obtained from repeated measurements and was about $3 \mu \mathrm{m}$ (one way) due to a combination of temperature drift in the interferometer and errors associated with resetting the micrometer. Alignment errors were judged to be negligible, since no changes could be observed on realignment of the optical components.

A major concern with our method of measuring group index has to do with variations of transmission properties along the length of the reference fiber. As a practical matter, we can only sample 
the properties of the fiber at either end, and make the assumption that the average value of $\mathrm{N}$ is representative of the entire length. The assumption of fiber uniformity has been examined in some detail for production fibers of the same type as used for the present standard reference fibers [4]. The authors find that the properties are indeed uniform within 0.02 percent. This sampling error constitutes the largest uncertainty in the group index.

Although the group index of dispersion-unshifted silica-based fibers undergoes a minimum in the wavelength interval around $1300 \mathrm{~nm}$, the wavelength dependence of the optical sources on measurements in this region is not entirely negligible. We account for this dependence by measuring the group index and time delay as close to the zero-dispersion wavelength as possible. Next we estimate the magnitude of the error which accrues if comparisons are made at wavelengths slightly different from $\lambda_{0}$.

The group index of the fiber can be expanded in a Taylor series about $\lambda_{0}$ as

$$
\left.\left.N(\lambda)=N\left(\lambda_{0}\right)+\frac{d N}{d \lambda}\right]_{\lambda 0}\left(\lambda-\lambda_{0}\right)+\frac{1}{2} \frac{d^{2} N}{d \lambda^{2}}\right]_{\lambda_{0}}\left(\lambda-\lambda_{0}\right)^{2}+\ldots
$$

where $\lambda_{0}$ is assumed to be known. Equation ( 8 ) can be written as

$$
\left.N(\lambda)-N\left(\lambda_{0}\right) \simeq \frac{1}{2} C \frac{d D}{d \lambda}\right)_{\lambda 0}\left(\lambda-\lambda_{0}\right)^{2}
$$

since $(d N / d \lambda)_{\lambda O}$ is proportional to the dispersion $D(\lambda)$ and is zero by definition. The constant $\left(\mathrm{d}^{2} \mathrm{~N} / \mathrm{d} \lambda^{2}\right)_{\lambda 0}$ is proportional to $\mathrm{dD} / \mathrm{d} \lambda$ and 
can be estimated from the fact that the Electronic Industries Association (EIA) recommendations restrict the value of the dispersion to less than $\pm 3.5 \mathrm{ps}(\mathrm{km}-\mathrm{nm})$ in the interval 1285 to $1350 \mathrm{~nm}$. This implies that $\mathrm{dD} / \mathrm{d} \lambda<2.4 \times 10^{-2} \mathrm{ps}\left(\mathrm{km}-\mathrm{nm}^{2}\right)$. Equation (9) then predicts that $N(\lambda)-N\left(\lambda_{0}\right)$ should be less than $1.0 \times 10^{-5}$ within $20 \mathrm{~nm}$ of $\lambda_{0}$.

\subsection{Pulse Time-of-Flight Errors}

Some of the uncertainties which can be identified with $\mathrm{T}_{d}$ include repeatability problems and inaccuracies in the digital delay generator. Most of the uncertainty in the time interval measurement comes from the limitations of the digital delay generator, and these are specific to the particular commercial instrument used in these investigations. An estimate of the magnitude of these errors may be obtained from the manufacturer's specifications and is approximately $0.9 \mathrm{~ns}$ for a $7 \mathrm{~km}$ fiber, i.e. a fractional uncertainty of $4.0 \times 10^{-5}$. This figure includes inherent instrumental error and jitter, along with effects due to aging and temperature stability.

Since we determine delay by visual alignment of an oscilloscope trace with a marker, there will be some error associated with the precise location of the pulse maximum. Because the pulse shape is approximately Gaussian, we can take this uncertainty to be 2 times the one sigma pulse duration (considering the two ends), or about $0.4 \mathrm{~ns}$.

Pulse distortion due to dispersion effects is totally negligible under these experimental conditions. The combined fractional uncertainty is about $4.2 \times 10^{-5}$. 


\subsection{Total Length Uncertainty for Long Fibers}

Using eq (1) and the propagation of error formula, we can estimate the total uncertainty in the length determination of a long fiber. For the $7 \mathrm{~km}$ SRF used in the present work we have $\Delta \mathrm{L}=2.0 \mathrm{~m}$, and $\Delta \mathrm{L} / \mathrm{L}=2.8 \times 10^{-4}$. These figures are dominated by group index uncertainties.

\section{OTDR Calibration}

One of the reasons for developing a standard reference fiber is to aid in the characterization and calibration of OTDRs. Along with attenuation, distance measurement accuracy is one of the principal quantities which is susceptible to accurate calibration in these devices [1]. The SRFs provided to contractors are also characterized for spectral attenuation; although, we do not discuss these measurements here.

An SRF can be combined with a fiber directional coupler to generate an infinite series of markers located precisely at $L / 2$ on the OTDR display. These recirculating delay line calibrators have been described in the literature $[12,13]$.

For calibration purposes only the time interval $T_{d}$ at the calibration wavelength need be specified accurately, since the OTDR does not measure length directly, only time interval. The reflectometer calculates length according to eq (1). Specifying the correct value for $T_{d}$, along with any group index $N$, is sufficient to establish the accuracy of the inferred values of length given by the OTDR. Therefore, the actual physical length of the SRF is immaterial. The correct value of $\mathrm{N}$ is required in determination by the OTDR of the actual physical length of another fiber. This point is important when establishing the errors associated with distance 
measurement accuracy. Since $\mathrm{T}_{d}$ can be measured much more accurately than $N$, the group delay uncertainties determine calibration accuracy for OTDR distance measuring capabilities.

\section{Conclusion}

We have described techniques for measuring two transmission properties of interest in standard reference fibers: group index of refraction and group delay. The asymmetric scanning Michelson interferometer has been demonstrated to be a simple, reliable, and accurate instrument for determining group index in small fiber samples. The estimated accuracy of these measurements, primarily limited by sampling errors, is about 0.03 percent. The group delay in a $7 \mathrm{~km}$ fiber was about $1.4 \mathrm{~ns}$ or 0.004 percent. This implies that the length of $7 \mathrm{~km}$ fiber can be determined with an overall uncertainty of about $2.0 \mathrm{~m}$. However, the only important quantity for calibrations of OTDR distance measurement accuracy is the time delay of the SRF, so these calibrations can be performed with an accuracy of about 0.004 percent. Using the same experimental techniques with better equipment, the group delay accuracy can probably be improved at least an order of magnitude.

\section{References}

[1] Danielson, B. L., "Calibration and standardization issues for the optical time-domain reflectometer." Nat. Bur. Stand. (U.S.) NBSIR 87-3078; 1987 December.

[2] Cohen, L. G. and Stone, J., "Interferometric measurements of minimum dispersion spectra in short lengths of single-mode fiber." Electron. Lett. 18(13): 564-566; 1982 June 24.

[3] Product information PI-115, Effective group index of refraction for SMF-21 Optical Fiber. Corning Glass Works. Corning, New York 14831. 
[4] Saunders, M. J. and Gardner, W. B., "Interferometric determination of dispersion variations in single-mode fibers." J. Lightwave Technol. LT-5 (12): 1701-1705; 1987 December.

[5] Gardner, W. B., "Dispersion measurements of single-mode and multimode optical fibers." Proc. of SPIE, Vol. 559: 18-23; 1985 August.

[6] Danielson, B. L. and Whittenberg, C. D., "Guided-wave reflectometry with micrometer resolution." Appl. Opt. 26(14); 2836-2842: 1987 July 15.

[7] Bell, R., Introductory Fourier transform spectroscopy. New York, NY: Academic Press; 1972. 382 p.

[8] Chamberlain, J. E., The principles of interferometric spectroscopy. New York, NY: John Wiley; 1979.

[9] Wilner, K. and van den Heuvel, A. P., "Fiber optic delay lines for microwave signal processing." Proc. IEEE 64(5): 805-807; 1976 May.

[10] Jackson, K. P., Newton, S. A., Moslehi, B., Tur, M., and Cutler, C. C., IEEE Microwave Theory Tech. MTT-33: 193-210; 1985 March.

[11] Newport Research Corporation Model F-IM1. This commercial product is mentioned in order to completely describe the experimental procedures, and does not imply endorsement. Other products may be equally suitable.

[12] Danielson, B. L., "Optical time-domain reflectometer specifications and performance testing." Appl. Opt. 24(15): 2313-2322; 1985 August 1.

[13] Newton, S. A., Nazarathy, M., and Trutna, W. R., "Measured backscatter signature of a fiber recirculating delay line." Appl. Opt. 25(12): 1879-1881; 1986 June 15. 


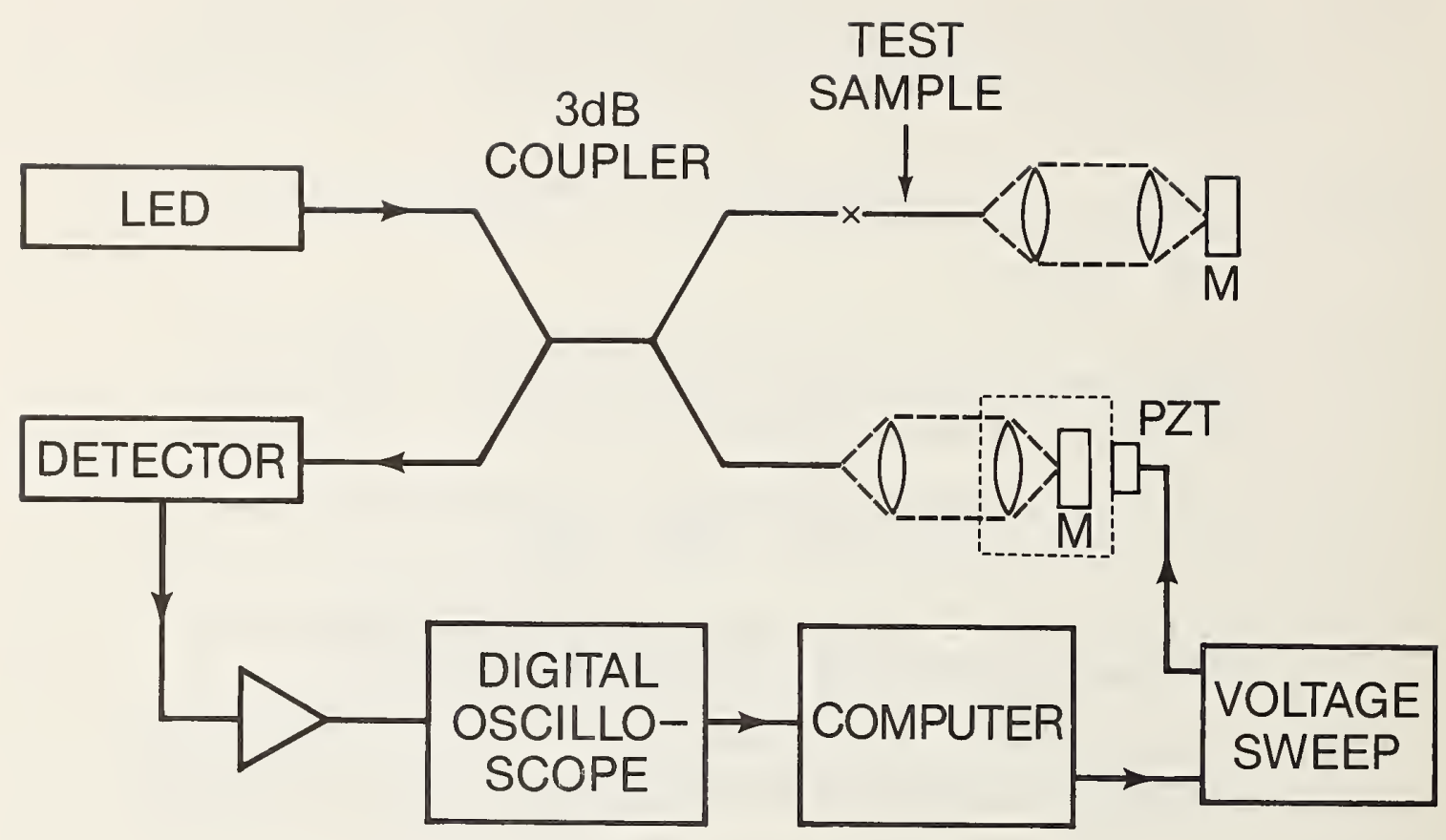

Figure 1. Experimental setup for measurement of group index with the asymmetric scanning Michelson interferometer. The cat's eye reflector is used in the test arm only to facilitate alignment of the test fiber in the vacuum chuck. 

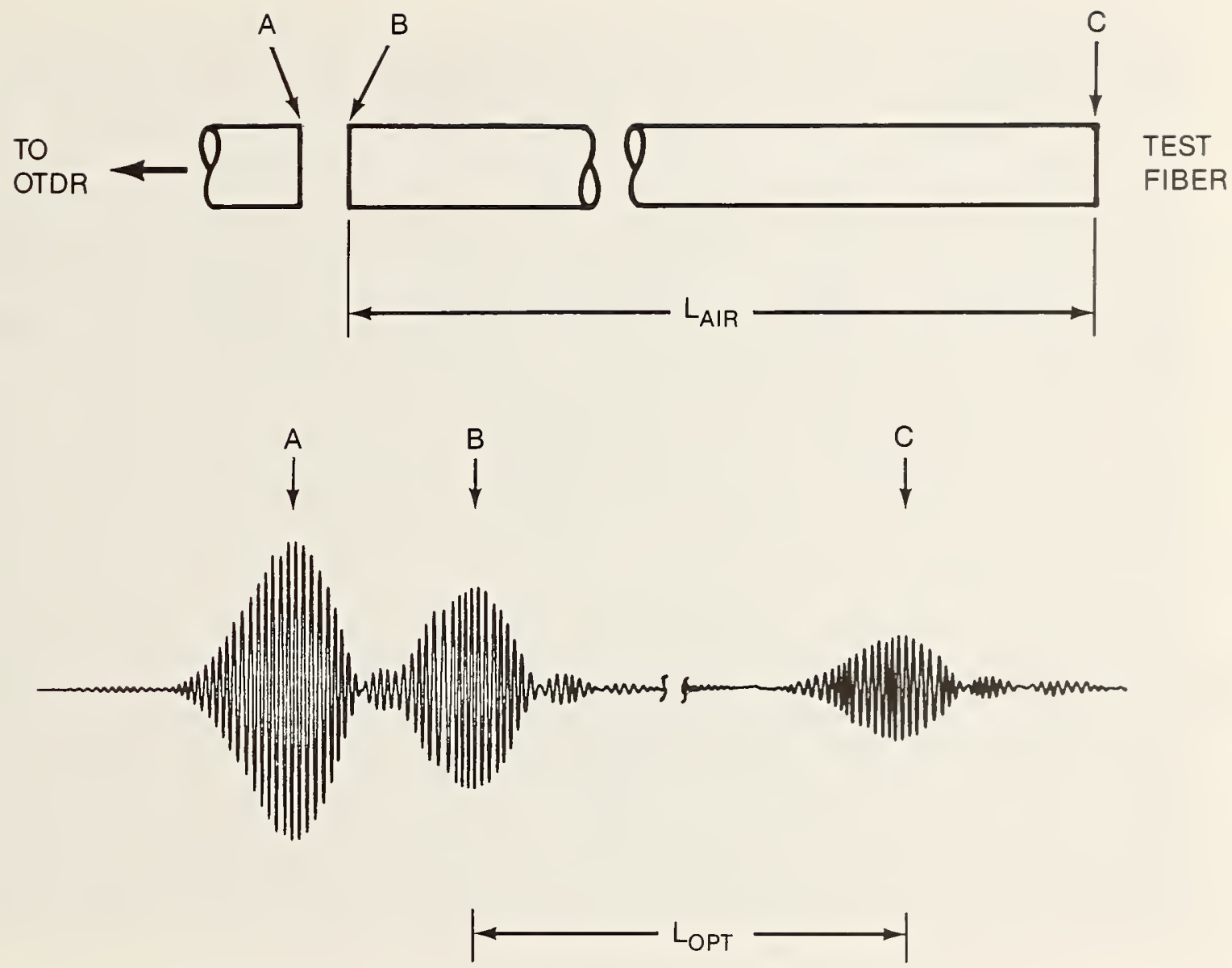

Figure 2. Typical interferograms (B) and (C) are generated from Fresnel reflections at the two ends of the fiber sample. The interferogram at (A) is due to the reflection from the launch fiber and is not important in the present context. 


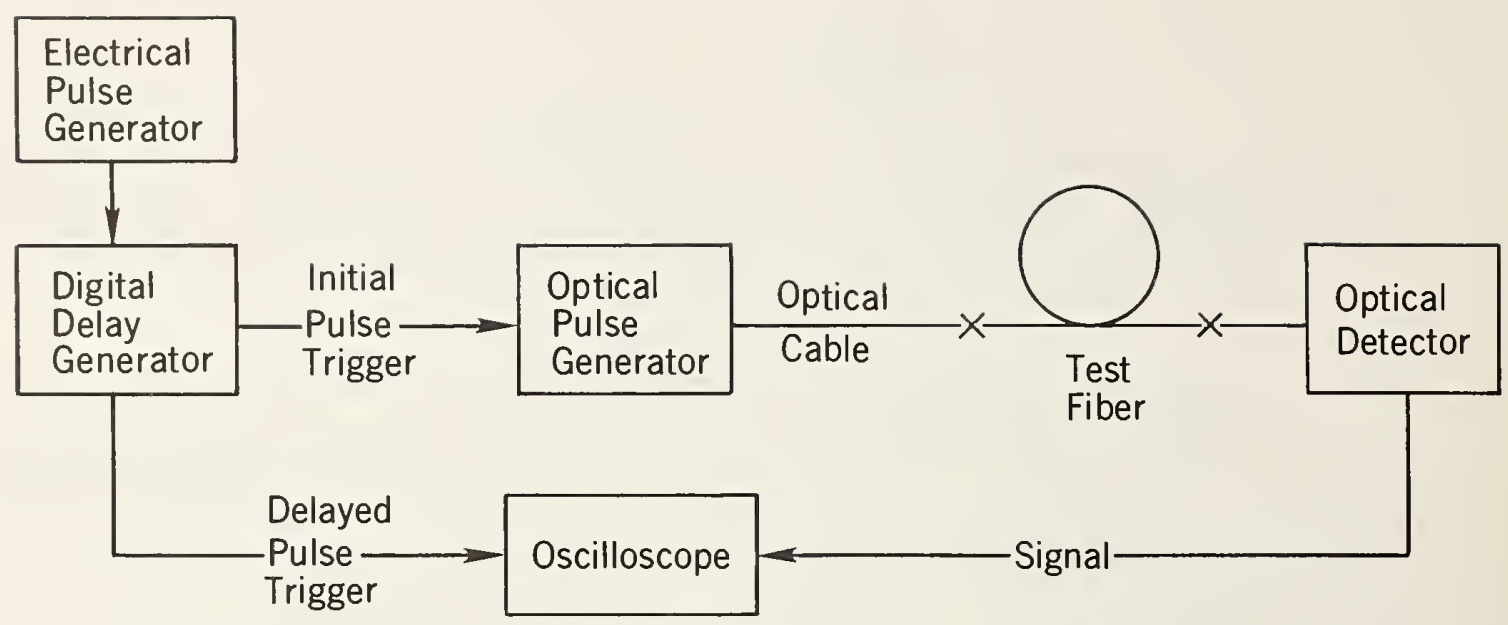

Figure 3. Experimental setup for measurement of time delay in the SRF. The electronic delay in the digital delay generator is made equal to the optical delay in the fiber. 


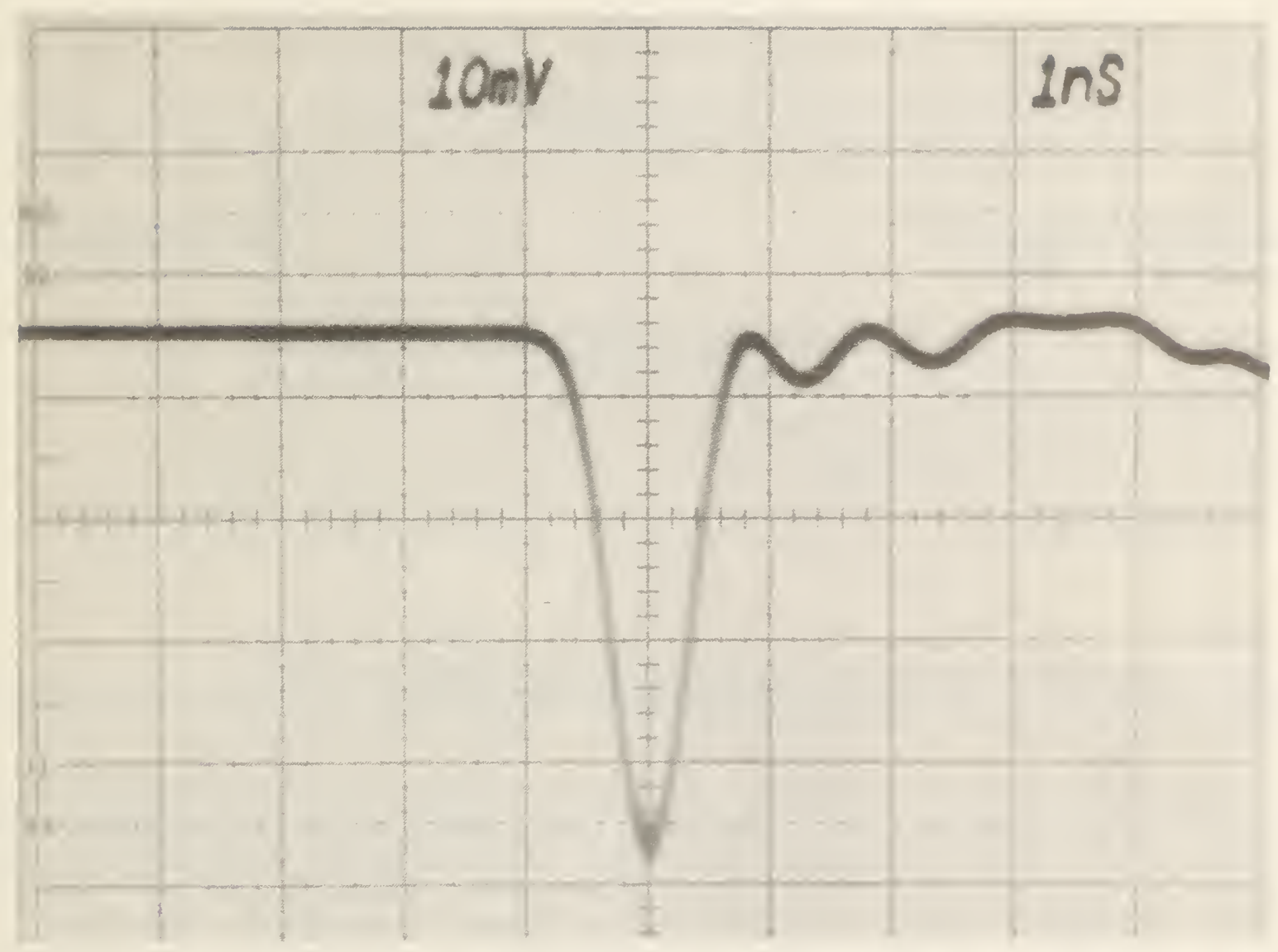

Figure 4. Profile of the optical pulse as displayed on the oscilloscope shown in figure 3 . 
NBS-114A IHEV, $2-A C$ )

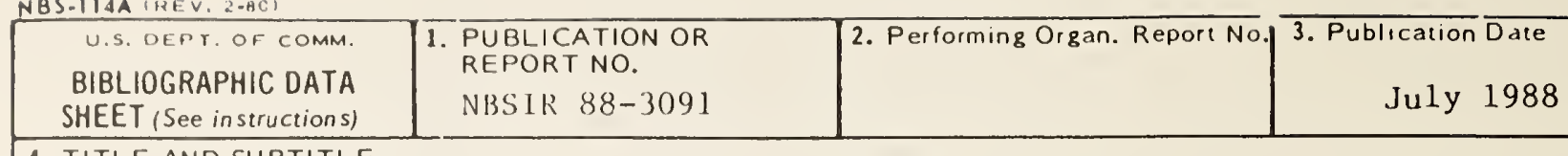

4. TITLE AND SUBTITLE

Group Index and Time Delay Measurements of a Standard Reference Fiber

5. AUTHOR(S)

B. L. Danielson and C. D. Whittenberg

6. PERFORMING ORGANIZATION (If joint or other than NBS, see instructions)

7. Contract/Grant No.

NATIONAL BUREAU OF STANDARDS

DEPARTMENT OF COMMERCE

WASHINGTON, D.C. 20234

9. SPONSORING ORGANIZATION NAME AND COMPLETE ADDRESS (Street, City, State, ZIP)

Department of the Navy

Naval Weapons Station/Seal Beach

Corona, California 91720-5000

10. SUPPLEMENTARY NOTES

Document describes a computer program; SF-185, FIPS Software Summary, is attached.

11. ABSTRACT (A 200-word or less factual summary of most significant information. If document includes a significant bibliography or literature survey, mention it here)

We describe measurement techniques for establishing a standard reference fiber with well characterized group index and time or group delay. Evaluation of an interferometric method indicates that fiber group index can be determined with a total estimated uncertainty of about 0.03 percent in smal1 samples. Group delay of the reference fiber was measured with an overall uncertainty less than 0.004 percent in a $7 \mathrm{~km}$ waveguide. We discuss the application of a standard reference fiber to calibration of the distance measurement accuracy of an optical time-domain reflectometer (OTDR).

12. KEY WORDS (Six to twelve entries; alphabetical order; capitalize only proper names; and separate key words by semicolons) calibration procedures; optical delay line; optical fiber group index; optical fiber group velocity; optical time-domain reflectometer; OTDR; standard reference fiber; etandard test fiber

13. AVAILABILITY

$\$$ Unlimited

$\square$ For Official Distribution. Do Not Release to NTIS $\square$ Order From Superintendent of Documents, U.S. Government Prinung Office, Washington, D.C.
20402 .

14. NO. OF PRINTED PAGES

20

15. Price

X] Order From National Technical Information Service (NTIS), Springfield, VA. 2216I 

